\title{
Separação e caracterização dos componentes de hemoglobina de Ptervgoplichthys pardalis, o acaribodó (*)
}

\author{
M. Brunori ('); J. Bonaventura $\left({ }^{2}\right) ;$ A. Focesi Jr. $\left({ }^{3}\right) ;$ M. I. Gaidames-Portus $\left({ }^{4}\right) ;$ M. T. Wilson $\left({ }^{5}\right)$
}

\section{Resumo}

Os quatro componentes de hemoglobina principais do hemolisado de Pterygoplichthys pardalis foram isolados e caracterizados. As propriedades funcionais investigadas para os componentes isolados compreendem o efeito de pH e ATP sobre: (i) o equilíbrio de $\mathrm{O}_{2}$, (ii) a cinética da dissociação de $\mathrm{O}_{2}$ (iii) a cinética da combinação de $\mathrm{CO}$. O componente I, corresponde a aproximadamente $50 \%$ da hemoglobina total, é caracterizado por propriedades funcionais que são diferentes das dos componentes II, III e IV, os quais são semelhantes. Assim, é provado, uma vez mais, que componentes múltiplos num hemolisado caem na categoria de hemoglobinas caracterizadas por propriedades funcionais distintas e complementares.

\section{INTRODUÇÃo}

Este artigo é um estudo dos componentes de hemoglobina de Pterygoplichthys pardalis, peixe da Bacia Amazônica pertencente à superordem Ostariophysi, ordem Cypriniformes, subordem Siluroidei (Greenwood et al., 1966; Fink, 1977). É um dos numerosos bagres que povoam as águas amazônicas e, provavelmente, o mais comum entre os mesmos.

A investigação das hemoglobinas concernente ao hemolisado do sangue de Pterygoplichthys foi efetuada pelas seguintes razões: (1) Entre os hemolisados investigados em alguns pormenores, durante a expedição do "Alpha Helix", a grande maioria, escolhida, por óbvias razões práticas, continha apenas um ou dois componentes hemoglobínicos. Um estudo cuidadoso de, pelo menos, um multicomponente do hemolisado parecia, portanto, essencial por ser necessário retirar conclu- sões relevantes a problemas fisiológicos. (2) Do ponto de vista da fisiologia respiratória, Pterygoplichthys é uma espécie muito interessante porque, além das guelras é provido de um sistema alternativo de troca de $\mathrm{O}_{2}$, associado aos seus intestinos, o que o torna um aeróbico opcional. Esta habilidade representa uma razão importante junto com sua estrutura coriácea, para a resistência incomum destas espécies. (3) É um peixe muito comum e abundante, que pode ser facilmente obtido em boa quantidade; além disso, ele representa, como um aeróbico opcional, um passo intermediário na evolução de organismos de vida aquática e terrestre. (4) Finalmente, é também um animal importante do ponto de vista comercial, sendo usado como alimento na região.

\section{MATERIAL E MÉTODOS}

A preparação da hemoglobina de sangue recém coletado seguiu o método de rotina (vide Antonini \& Brunori, 1971). A purificação foi feita por passagem através de coluna de Sephadex G-25 a pH 7,5, seguida por passagem através de coluna de troca iônica "mixed bed".

A eletroforese foi realizada em gel poliacrilamida. A separação dos componentes fơ feita em coluna cromatográfica de $D E A E$, da seguinte maneira: a coluna e o hemolisado foram equilibrados com Tris $0,05 \mathrm{M}$ pH 8,5; após aplicação da amostra, a coluna foi lavada com aproximadamente $200 \mathrm{ml}$ do mesmo tampão e posteriormente foi iniciada em gradiente de $\mathrm{pH}$ com $500 \mathrm{ml}$ de Tris $0,05 \mathrm{M} \mathrm{pH} 8,5$ e $500 \mathrm{ml}$ de Tris-acetato $0,05 \mathrm{M} \mathrm{pH} \mathrm{6,0.}$

(•) - Versão original inglesa publicada em Comp. Biochem. Physiol. vol. 62 A (t). 1979.

(1) - CNR Centre for Molecular Biology, Institutes of Chemistry and Biochemistry, Faculty of Medicine, University Rome, Rome, Italy.

(2) - Duke University, Marine Laboratory, Beaufort, N.C. 28516, U.S.A. Established Investigator of the American Heart Association.

(3) - Departamento de Bioquímica, I.B., Universidade Estadual de Campinas, 13100 Campinas, SP, Brasil.

(4) - Instituto Nacional de Pesquisas da Amazônia, 69000 Manaus, AM, Brasil.

( 5 ) - Department of Chemistry, University of Essex, Wivenhoe Park, Colchester, Essex, U.K. 
O equilíbrio de oxigênio foi medido segundo o método de Riggs \& Wolbach (1956).

Experimentos cinéticos foram efetuados em um aparelho "Stopped flow" Gibson-Durrum equipado com uma célula de observação de $2 \mathrm{~cm}$. A cinética da dissociação de oxigênio foi medida usando-se o método do ditionito (Antonini \& Brunori, 1971). Soluções de CO foram obtidas diluindo-se quantitativamente uma solução estoque de água destilada equilibrada com CO a $1 \mathrm{~atm}$ e $30 \pm 1^{\circ} \mathrm{C}$, conc. $=0,86$ $\mathrm{mM}$ (Tabelas Críticas Internacional, Washburn, 1926)

\section{RESUltadoS E DISCUSSÃo}

\section{COMPORTAMENTO ELETROFORÉTICO}

E CROMATOGRÁFICO

Os hemolisados de diversos indivíduos adultos foram caracterizados eletroforeticamente. As análises de, pelo menos, 20 espécimes provou que: (i) os hemolisados continham múltiplos componentes hemoglobínicos; (ii) a presença de uma banda principal, correspondendo a aproximadamente $50 \%$ da $\mathrm{Hb}$ total e 3 outros componentes menores; (iii) o padrão foi constante de um indivíduo para outro. Este padrão corresponde ao descrito por Fyhn et al. (1978) para espécies 1 de Pterygoplichthys.

A separação dos vários componentes foi efetuada por cromatografia em DEAE-Sephadex (ver métodos). A Fig. 1 ilustra o padrão de eluição, o qual é consistente com o padrão eletroforético observado. O componente principal (aqui denominado Componente I) foi eluído da coluna com o tampão inicial; após iniciar o gradiente, os outros componentes foram progressivamente eluídos. As frações dos 3 picos mais proeminentes (denominados, Componentes II, III e IV respectivamente) foram agrupados e coletados como mostra a Fig. 1. Estes 4 componentes principais foram submetidos a gel-eletroforese em acrilamida e mostraram-se homogêneos.

Outros componentes ainda menores foram observadős no cromatograma, mas parecem constituir uma pequena fração da hemoglobina total e foram, portanto, ignorados.



Fig. 1 - Cromatografia em DEAE - Sephadex do hemolisado de Pterygoplichthys (espécime n. ${ }^{\circ} 772$ ). As fraçōes indicadas foram agrupadas e cienominadas de I a IV em ordem de eluição (da esquerda para a direita). A gradiente $(500 \mathrm{ml}$ de tris-HCI $0,05 \mathrm{M} \mathrm{pH} 8,5+500 \mathrm{ml}$ tris-acetado $0,05 \mathrm{M} \mathrm{pH}$ $6,0)$ foi iniciado após aproximadamente 40 frações coletadas.

\section{PROPRIEDADES DA UNIÃO DE OXIGÊNIO}

A curva de equilíbrio de $\mathrm{O}_{2}$ do Componente I, sob condições de fracionamento é claramente cooperativa, com um valor do parâmetro de Hill - $n$, abrangendo de 1,9 a $\mathrm{pH} 6,7$ a 1,3 a pH 8,5. Como mostra a Fig. 2, o componente I fracionado mostra um pequeno efeito Bohr reverso centralizado em cerca de $\mathrm{pH} 7,2$ a $25^{\circ} \mathrm{C}$. Deve-se notar que estes experimentos foram efetuados na presença de $1 \mathrm{mM}$ de EDTA para diminuir a velocidade de auto-oxigenação a baixos valores de $\mathrm{pH}$. O efeito do ATP (1 mM) é insignificante a $\mathrm{pH}>7,5$; a $\mathrm{pH}$ mais baixa, a adição de ATP é associada com um decréscimo na afinidade de $\mathrm{O}_{2}$, bem como um leve decréscimo em $n$ (por ex: a pH 6,7 $n$ vai de 1,87 a 1,4 por adição de $1 \mathrm{mM}$ de ATP). A Fig. 3 mostra a dependência da concentração total de ATP de $\log \mathrm{P}_{1 / 2}$ medida para o Componente I a $\mathrm{pH} 6,2$, em bis-tris $0,05 \mathrm{M}$ e $20^{\circ} \mathrm{C}$. Os dados mostram que este $\mathrm{pH}$ efeito total de ATP é deduzido a concentrações consideravelmente maiores que $1 \mathrm{mM}$. 
As propriedades da união de $\mathrm{O}_{2}$ do componente IV fracionado (na presença de $1 \mathrm{mM}$ EDTA) foi investigada em um intervalo de $\mathrm{pH}$ de 6,2 a 8,4 . A Fig. 2 mostra o efeito Bohr para este componente sob condições de fracionamento, bem, como na presença de ATP $(1 \mathrm{mM})$. Os dados mostram que, em contraste ao comportamento I, a dependência de $\mathrm{pH}$ da afinidade de $\mathrm{O}_{2}$ é, na verdade, muito marcante, o $\left(\Delta \log P_{1 / 2} / \Delta \mathrm{pH}\right)$ sendo aproximadamente 1 abaixo de 7 . O valor de $n$ é sempre abaixo de 2, abrangendo de 1,1 a 1,7 e mostra obscura dependência de $\mathrm{pH}$ ou ATP.

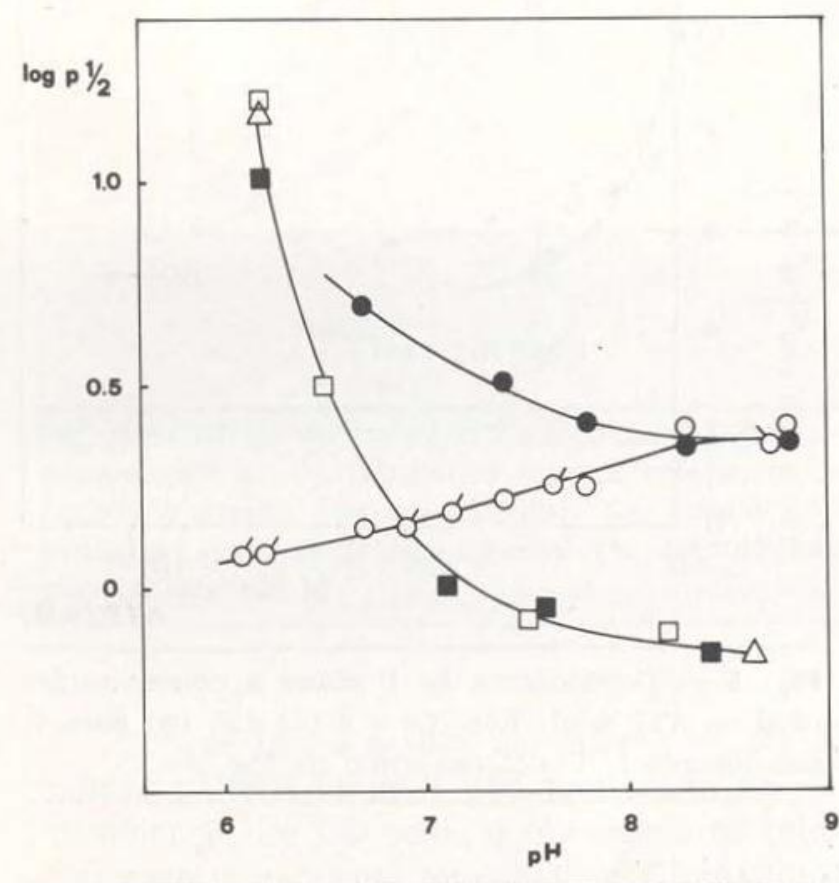

Fig. 2 - Efeito Bohr de oxigênio dos componentes de Pterygoplichthys. O componente I é indicado por círculos: O, fracionado; $+1 \mathrm{mM}$ ATP, of indica experimentos efetuados a $20^{\circ} \mathrm{C}$ e deslocados de 0,05 nas escalas de $\log p_{1 / 2}$. O componente IV é indicado por quadrados $\square$, fracionado; $+1 \mathrm{mM}$ ATP. O componente III é indicado por triângulos $\Delta$. Condiçōes: bis-tris $0,05 \mathrm{M}$ ou tampão tris + $1 \mathrm{mM}$ EDTA e $25^{\circ} \mathrm{C}$. Concentraçäo de $\mathrm{Hh} \sim 1$ $\mathrm{mg} / \mathrm{ml}$.

O componente III fracionado parece ter essencialmente a mesma afinidade $\operatorname{com~} \mathrm{O}_{2}$ que o componente IV. É muito significativo que a afinidade pelo $\mathrm{O}_{2}$ do componente IV, embora depen̂dente de $\mathrm{pH}$, é independente de ATP, mesmo na região de baixo $\mathrm{pH}$ onde ATP tem um efeito no comportamento do componente I.
Nossos resultados ampliam o trabalho de Weber et al. (1978), que isolaram duas fraçōes hemoglobínicas, $a$ e $b$, do hemolisado de Pterygoplichthys por focalização do ponto isoelétrico. Parece que a fração $b$ (Fig. 4B de Weber et al., 1978) contém ambos os componentes IV e outros componentes com propriedades mais semelhantes às do componente $\mathrm{I}$.

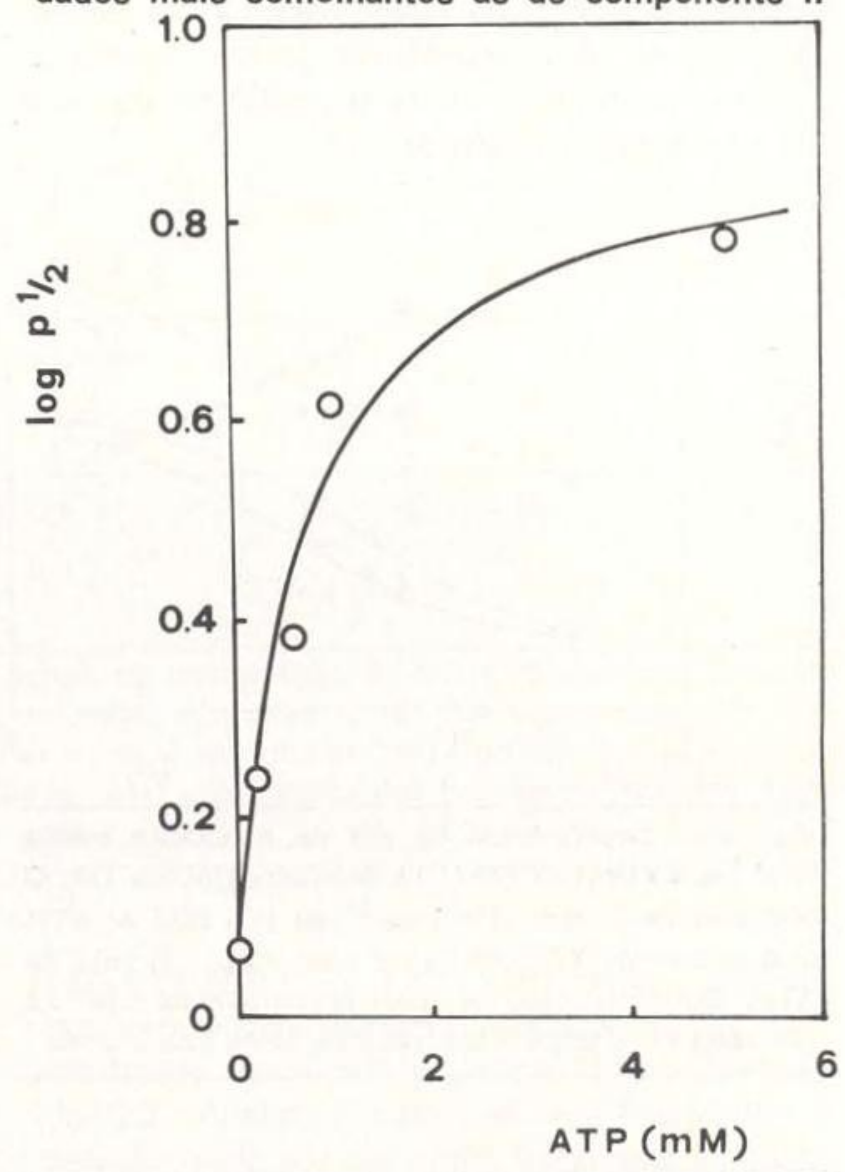

Fig. 3 - Dependência de $\log \mathrm{p}\left(\mathrm{O}_{2}\right)$ na concentração total de ATP para o componente I. Condições: $\mathrm{pH} 6,7 \mathrm{em}$ bis-tris $0,05 \mathrm{M}$ e $20^{\circ} \mathrm{C}$.

\section{PROPRIEDADES CINÉTICAS}

a. Combinação com monóxido de carbono

Esta reação foi estudada em paralelo com todos os 4 componentes (a) sob condições de fracionamento, (b) como uma função da concentração de $\mathrm{CO}$ e (c) a dois $\mathrm{pHs}(\sim 5,6$ e $7,7)$. Em ambos os valores de $\mathrm{pH}$, descobriuse que a total pseudo constante média de $1^{\text {a }}$ ordem cresce linearmente com a concentração ligante ( $\mathrm{CO}$ abrangendo de 7 a $43 \mu \mathrm{M}$, concentração de $\mathrm{Hb} \sim 2,5 \mu \mathrm{M}$ (em heme). Estes 
experimentos mostraram que, a ambos valores de $\mathrm{pH}$, os componente II, III e IV são semelhantes, enquanto o componente I é diferente dos outros três.

A Fig. 4 representa a dependência de $\mathrm{pH}$ da total constante média de combinação de $2^{\mathrm{a}}$ ordem para CO (I'), ambos na ausência e presença de ATP $(1,25 \mathrm{mM})$, para os componentes I e IV. A concordância destes resultados com o descoberto sobre o equilíbrio de $\mathrm{O}_{2}$ é imediatamente evidente.



Fig. 4 - Dependência de $\mathrm{pH}$ da constante média total para a ligação $C O$ (I') à deoxiemoglobina (s). 0 componente $\mathrm{I}$, sem $(\mathrm{O})$ e com (•) $1,25 \mathrm{mM}$ de ATP. O componente IV $\operatorname{sem}(\Delta)$ e com $(\Delta) 1,25 \mathrm{mM}$ de ATP. Concentração de $\mathrm{Hb}$ aproximadamente 2,5 $\mu \mathrm{M}$ (heme). Tampão bis-tris $0,05 \mathrm{M}$ ou tris e $20^{\circ} \mathrm{C}$.

O componente cinético do componente I fracionado é independente de $\mathrm{pH}$ e mostra um efeito de ATP apenas na região de $\mathrm{pH}$ ácido. Assim, a pH 5,5 a 6,0 a constante média de combinação de CO decresce com adição de ATP, como mostra a Fig. 5, a qual representa a dependência de concentração de ATP de I' para componente $\mathrm{I}$ a dois valores de $\mathrm{pH}$. Deve-se notar que o curso de tempo de combinação de $\mathrm{CO}$ é um tanto homogênea $\left(2^{\mathrm{a}}\right.$ ordem) ou algo autocatalítico, dependendo do $\mathrm{pH}$ e (ou) da presença de ATP.

No caso do componente IV, o curso de tempo de combinação de $\mathrm{CO}$ é essencialmente homogênea a valores de $\mathrm{pH}$ abaixo de 7 , enquanto parece ser algo heterogêneo a $\mathrm{pH}>7$. A dependência de $\mathrm{pH}$ de I', segue um efeito
Bohr normal, trocando aproximadamente 3,5 vezes baixando de $\mathrm{pH} 5,5$ para 7,7 (Fig. 4). Ainda mais importante é a descoberta de que a adição de ATP $(1,25 \mathrm{mM})$ tem um efeito muito pequeno sobre $\mathrm{I}$ ' a todos os $\mathrm{pH}$ examinados. A presença de um mensurável efeito cinético Bohr, a (quase completa) ausência de efeito de ATP e a similaridade no comportamento cinético dos componentes II, III e IV, estão todos em consonância com o equilíbrio verificado e referido acima.

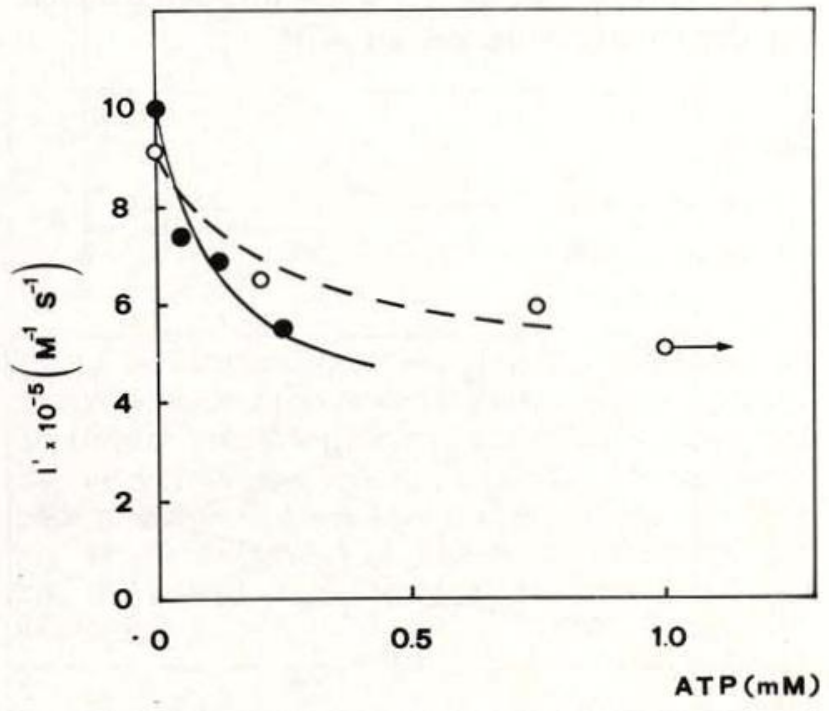

Fig. 5 - Dependência de I' sobre a concentração total de ATP a pH 5,55 (O) e a pH 6,21 (•) para o componente I. Condições como na Fig. 4.

Um número limitado de experimentos de "flash photolysis" foi feito com o componente $\mathrm{I}$, a dois valores de $\mathrm{pH}$. Alguns dos dados experimentais são citados na Fig. 6. Dois pontos se evidenciam do exame destes experimentos: (i) em fotólise total na presença e ausêricia de ATP, a reação é claramente autocatalítica e não há evidência de uma rápida forma reagente dada a presença de dimeros em solução, em contraste com o observado para a $\mathrm{HbA}$ (Antonini \& Brunori, 1971; (ii) em fotólise parcial (aproximadamente $10 \%$ de fotodissociação), a combinação com CO é muito mais rápida que em fotólise total. Esta é uma indicação direta da existência de uma rápida espécie reagente, o qual é $\sigma$ único estado significativamente populado a baixos níveis de fotólise (ex., à altas saturações de $\mathrm{CO}$ ) . 


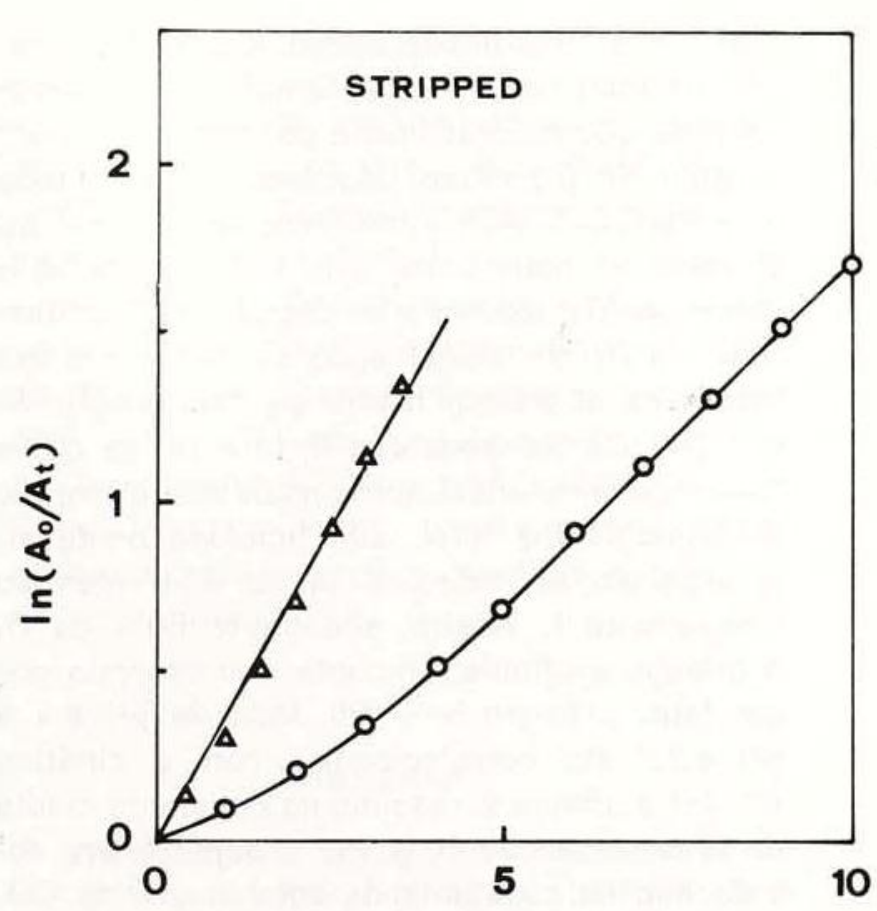

Time (m sec)

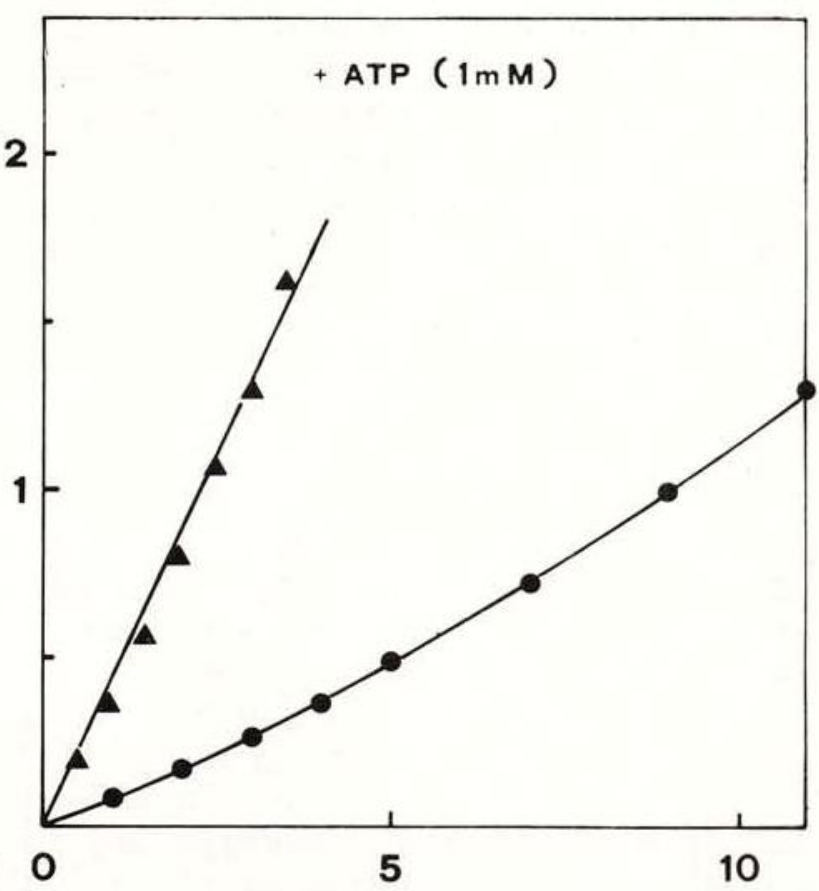

Time (m sec)

Fig. 6 - Efeito da extensão de fotólise no curso de tempo de combinação de CO a desoxiemoglobina do componente I. Os resultados citados referem-se a experimentos efetuados a pH 5,45 sem e com ATP (esquerda e direita respectivamente). Os resultados da fotólise total são mostrados como círculos (abertos ou fechados) e os de fotólise parcial ( $\%$ de fotodissociação $=10 \%$ ) como triângulos (aberto ou fechado). Tampão bis-tris $0,05 \mathrm{M}$.

Nota-se que a ambos os valores de $\mathrm{pH}$, a adição de ATP (1 $\mathrm{mM})$ não tem efeito na recombinação de CO como o observado na fotólise parcial, enquanto tem algum efeito na fotólise completa (vide fig. 6). Esta verificação está em concordância com os resultados citados acima (vide fig. 4).

\section{b. Dissociação de oxigênio}

A dissociação de $\mathrm{O}_{2}$ pelo método do ditionito foi investigado para todos os componentes em paralelo, a pH $\sim 5,6$ e 7,7 na ausência de ATP sob condições "fracionamento". Em todos os casos, o curso de tempo de dissociação de $\mathrm{O}_{2}$ foi de $1^{\text {a }}$ ordem por uma boa aproximação. Novamente foi verificado que os componentes II, III e IV tem constantes médias muito similares, enquanto o componente I é diferente dos outros três.

A fig. 7 mostra a dependência de $\mathrm{pH}$ da constante média de dissociação de $\mathrm{O}_{2}(\mathrm{~K})$ para os componentes I e III, na ausência e na pre- sença de ATP $(1,25 \mathrm{mM})$. Novamente o comportamento é consistente tanto com o equilibrio verificado como com a cinética da combinação de CO. Assim, o componente I fracionado é caracterizado por um efeito Bohr reverso muito pequeno, e a adição de ATP aumenta significativamente a constante "off" a pH abaixo de 6,5 (um efeito nítido é notado nos experimentos paralelos a pH 5,6-5,7). O comportamento III mostra um efeito Bohr positivo muito forte (abaixo de $\mathrm{pH} 7$ ), o valor de $K$ aumentando de um fator de $\sim 10$, indo de $\mathrm{pH} 7$ a pH 5,3. Em adição, não foi observado efeito de ATP na cinética da dissociação de $\mathrm{O}_{2}$ do componente III.

\section{Conclusões}

Diversas espécies de bagres foram examinadas durante a Expedição "Alpha Helix" (Fink \& Fink, 1978; e outros artigos deste volume), mas na maioria dos casos a atenção foi concentrada nos caracterizados por hemolisados 
que continham um (Pseudodoras e Brachyplatystoma) ou dois (Hoplosterum) componentes hemoglobínicos. Pterygoplichthys pardalis foi o único caso com 4 componentes principais, cuidadosamente estudado, e como tal ele pode desempenhar um papel importante nos padrōes de entendimento de comportamento funcional de hemoglobinas de bagres da Amazônia.

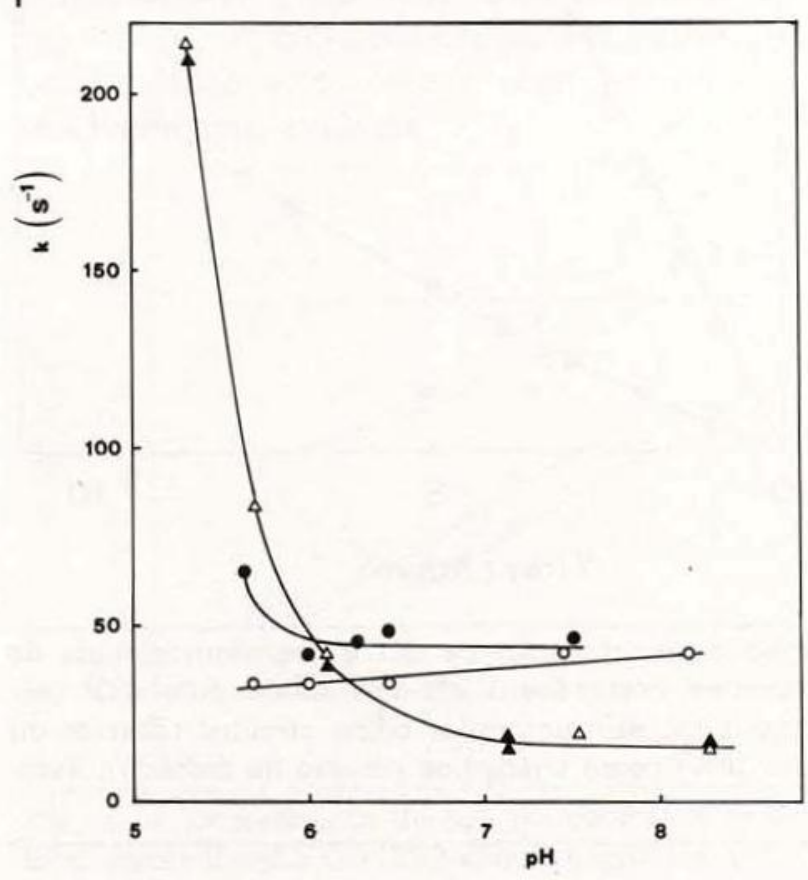

Fig. 7 - Efeito de pH na constante média da dissociação de $\mathrm{O}_{2}(\mathrm{~K})$ para componente $\mathrm{I}$ na ausência (O) e na presença ( ) de $1,25 \mathrm{mM}$ ATP e componente III na ausência $(\Delta)$ e na presença ( $\Delta$ ) de 1,25 mM de ATP. Tampões de bis-tris $0,05 \mathrm{M}$ ou tris e $20^{\circ} \mathrm{C}$. Concentraçōes de $\mathrm{Hb} \sim 25 \mu \mathrm{M}$ (heme).

As principais descobertas e conclusões deste trabalho podem ser resumidas como se segue: (i) os quatro maiores componentes hemoglobínicos podem ser divididos, funcionalmente, em dois grupos: um representado pelo componente $\mathrm{I}$ e o outro compreendendo os componentes II, III e IV. Assim, dentro da precisão de nossas medidas, os três componentes são, na verdade, muito, se não idênticos, no equilíbrio e cinética da reação $\mathrm{com} \mathrm{O}_{2}$ e $\mathrm{CO}$ (ii). Em geral, os experimentos de equilíbrio estão em excelente (semi-quantitativo) harmonia com os resultados cinéticos. Esta conclusão confirma ambos os efetores alostéricos, ex. prótons e ATP. (iii) O componente I, que representa aproximadamente metade da hemoglobina total presente no hemolisado, é caracterizado quando fracionado por um efeito Bohr reverso de $\mathrm{O}_{2}$, muito pequeno; sua afinidade total com $\mathrm{O}_{2}$ é ATP-dependente apenas nos intervalos de mais baixo $\mathrm{pH}$. Cineticamente, o efeito de ATP resulta num decréscimo na constante média de combinação de $\mathrm{CO}$ e um aumento na constante média da dissociação de $\mathrm{O}_{2}$. (iv) Os componentes II, III e IV, os quais juntos compreendem aproximadamente metade da hemoglobina total, são funcionalmente similares um ao outro e muito diferentes do componente I. Assim, seu efeito Bohr de $\mathrm{O}_{2}$ é grande, a afinidade ligante decrescendo por um fator próximo a $\sim 20$, indo de $\mathrm{pH} 8,5$ a $\mathrm{pH} 6,2$. Isto correlaciona-se com a cinética em (a) o grande acréscimo na constante média da dissociação de $\mathrm{O}_{2}$ e (b) o significativo decréscimo na constante de cơmbinação de $\mathrm{CO}$, com o decréscimo do $\mathrm{pH}$. Em adição, o efeito de ATP é desprezível sobre o intervalo de $\mathrm{pH}$ examinado. ( $v$ ) Estes resultados somados juntos nos levam a concluir que os vários componentes de hemoglobina de Pterygoplichthys caem em duas categorias caracterizada por propriedades funcionais que são complementares com respeito à influência de típicos efe. tores alostéricos. Isto sugere que: (a) a presença de múltiplos componentes com propriedades funcionais altamente diferentes é um padrão comum, embora não universal, em sangue de peixe e (b) embora não se possa oferecer uma explicação total no presente, nós consideramos igualmente uma relação, ainda a ser definida, entre propriedades funcionais e papel fisiológico destas hemoglobinas. Tal relação foi proposta e substanciada para as hemoglobinas de truta (Salmo irideus) (Brunori, 1975). A existência de subgrupos de hemoglobinas com funções complementares parece, portanto, razoavelmente geral, pois tem se verificado também em outras espécies estudadas a bordo do "Alpha Helix", por ex. para o sistema de 2 componentes de Mylosomma (Martin et al., 1978).

Esta complementaridade de funçōes adquiriria importância se fosse verificado que os vários componentes coexistem num mesmo eritrócito, como mostra ser o caso do sangue de truta (Brunori et al., 1974) . 


\section{AGRAdECIMENTOS}

Este trabalho foi financiado por National Science Foundation Grant PCM 75-06451. Somos gratos ao Governo Brasileiro pela permissão de o "Alpha Helix" percorrer águas do Amazonas. M. Brunori expressa seus agradecimentos a National Research Council (CNR) da Itália pela cobertura financeira para a viagem. M. T. Wilson agradece os fundos para a viagem recebidos da Royal Society, U. K. e Duke University, USA. A. Focesi Jr. foi parcialmente assistido pelo Conselho Nacional de Ciência e Tecnologia.

\section{SUMMARY}

The four main hemoglobin components of the hemolysate of Pterygoplichthys pardalis have been isolated and characterized. The functional properties investigated for the isolated components comprise the effect of $\mathrm{pH}$ and ATP on (i) the $\mathrm{O}_{2}$ equilibrium, (ii) the $\mathrm{O}_{2}$ dissociation kinetics, (iii) the $\mathrm{CO}$ combination kinetics. Component I, corresponding to approximately $50 \%$ of the total hemoglobin, is characterized by functional properties which are distinctly different from those of Components II, III and IV, which are alike. Thus it is shown, once more, that multiple components in an hemolysate fall into categories of hemoglobins characterized by distinct and complementary functional properties.

\section{BIBLIOGRAFIA}

ANTONINI, E. \& BRUNORI, M.

1971 - "Hemoglobin and myoglobin in their reactions with ligands", North Holland, Amsterdam.

BRUNORI, M.

1975 - Molecular adaptation to physiological requirements. Curr. Top. Cell. Regulation, 9:1-39.
Brunori, M.; Giardina, B.; ANtonini, E.; Benedetti, P.A. \& BIANCHINI, G.

1974 - J. Mol. Biol., 86:165-169.

BUNN, H.F. \& RIGGS, A.

1978 - A medida do efeito Bohr em hemoglobinas de peixe por focalização elétrica em gel. Acta Amazonica 8(4): Suplemento. (Este volume).

FINK, W.L. \& FINK, S.V.

1978 - A Amazônia Central e seus peixes. Acta Amazonica 8(4): Suplemento. (Este volume).

FYHN, U.E.H.; FYHN, H.J.; DAVIS, B.J.; POWERS, D.A.;

FINK, W.L. \& GARLICK, R.L.

1978 - Heterogeneidade de hemoglobina nos peixes da Amazônia. Acta Amazonica 8(4) : Suplemento. (Este volume).

Greenwood, P.H.; Rosen, D.E.; WeItZMAN, S.H. \& MYERS, G.S.

1966 - Phyletic studies of teleostean fishes with a provisional classification of living forms. Bull. Am. Mus. Nat. Hist., 131:339-455.

MARTIN, J.P.; BonaVentura, J.; BRUNori, M. ; Fyhn, H.J.; FYhN, U.E.H.; Garlick, R.L.; POWERS, D.A. \& WILSON, M.T.

1978 - Isolamento e caracterização dos componentes da hemoglobina de Milossoma sp., um teleósteo da Amazônia. Acta Amazonica 8(4): Suplemento. (Este volume).

RIGGS, A.F. \& WоLBACH, R.A.

1956 - Sulfhydryl groups and the structure of hemoglobin. J. Gen. Physiol., 39:585-605.

WASHBURN, E.W.

1926 - International critical tables of numerical data, Physics, Chemistry and Technology.

WEBER, R.E. \& WoOD, S.C.

1978 - Efeito de nucleosídio trifosfato eritrocítico no equilíbrio de oxigênio de hemoglobinas completas e fracionadas de Hypostomus e Pterygoplichthys, bagres aeróbicos facultativos. Acta Amazonica 8(4) : Suplemento. (Este volume). 